Tohoku J. Exp. Med., 1990, 161, 55-64

\title{
Cosinor Analysis of Changes in Circadian Blood Pressure Rhythm with Aging in Spontaneously Hypertensive Rats
}

\author{
Masanori Munakata, Yutaka Imai, Naoyoshi Minami, \\ Syuuichi Sasaki, Toshiyuki Ichijyo, ${ }^{*}$ Makoto \\ Yoshizawa, ${ }^{*}$ Hiroshi Sekino, $†$ Keishi Abe and Kaoru \\ Yoshinaga \\ The Second Department of Internal Medicine, Tohoku \\ University School of Medicine, * Department of Technology, \\ Tohoku University, Sendai 980 and †Kojin-kai Hospital, \\ Sendai 980
}

Munakata, M., Imai, Y., Minami, N., Sasaki, S., Ichijyo, T., Yoshizawa, M., Serino, H., Abe, K. and Yoshinaga, K. Cosinor Analysis of Changes in Circadian Blood Pressure Rhythm with Aging in Spontaneously Hypertensive Rats. Tohoku J. Exp. Med., 1990, 161 (1), 55-64_Evolution of circadian rhythm of blood pressure with age in male spontaneously hypertensive rats (SHR) was compared with that in male Wistar Kyoto rats (WKY). Three different age groups (5-6 week old, 19-22 week old, 29-31 week old) were provided for each strain. Chronogram and cosinor method were used for time series analyses. In all age groups of WKY, a diurnal fall and a nocturnal rise in blood pressure (BP) as well as in heart rate (HR) were observed. There was no significant difference in acrophase between BP and HR in each age group of WKY. Regarding SHR, however, each age group demonstrated a different relation between circadian BP rhythm and HR rhythm. In 5-6 week old SHR, a difference in acrophase between $\mathrm{BP}$ and HR (about $6.9 \mathrm{hr}, p<0.1$ ) was observed, which became more conspicuous with the increase in age, eventually presenting an inverted relation between BP and HR in 19-22 week old SHR. The relation between circadian BP rhythm and circadian HR rhythm in 29-31 week old SHR was almost identical with that in 1922 week old SHR. In other words, a phase lag of acrophase of BP from that of HR already observed in young SHR increased with aging. The results indicate that parasympathetic periodicity remained unchanged even in SHR since circadian HR rhythm was similar in all groups, while the periodicity of sympathetic neural tone relating to the regulation of circadian $\mathrm{BP}$ rhythm seems to be disturbed. The mechanism responsible for development and maintenance of high BP in SHR may be linked to a disturbance in the sympathetic mechanism which regulates BP periodicity. circadian rhythm; blood pressure; rats; cosinor method; hypertension

It is well known that human blood pressure (BP) demonstrates a large

Received April 23, 1990 ; revision accepted for publication April 28, 1990. 
rhythmic (Richardson et al. 1964 ; Bartter et al. 1976 ; Michael et al. 1978) as well as random variation (Weber et al. 1982). It is also reported that in human beings, physical activity has episodic but systematic effects on BP (Rowlands et al. 1980). Though circadian rhythm of BP is assumed to comprise two stages, i.e., higher BP while awake and lower BP while asleep, there is no clear explanation for this rhythm. Mancia et al. (1983) stated that a circadian variation of BP is considerably affected by the central nervous system activity. Littler et al. (1975) advocated that the nocturnal fall in BP is secondarily caused by sleep. Change of posture is also cited as an essential factor for changes in circadian BP rhythm (Richards et al. 1986). However, no reduction in BP during sleep has been noted in several disease states (Redman et al. 1976 ; Mann et al. 1983; Imai et al. 1988). This fact is cited against the hypothesis that circadian BP rhythm is affected by physical activity or the length and depth of sleep.

It has been reported that the inherent oscillator, suprachiasmatic nucleus (SCN) of the anterior hypothalamus, mediates various physiological circadian rhythms such as body temperature, sleeping and waking, and heart rate $(\mathrm{HR})$ in rats (Moore 1983). Although the neural mechanism by which SCN regulates circadian rhythm is yet to be clarified, a vasopressinergic neural mechanism may be postulated as one such regulatory mechanism, since the largest population in peptidecontaining neurons in rat SCN is vasopressin containing neurons (Moore 1983). Spontaneously hypertensive rats (SHR), a model of human hypertension, are known to have many central abnormalities including the vasopressinergic system (Rascher et al. 1982 ; Morris et al. 1983). This leads to a question as to whether SHR has some alteration of circadian cardiovascular rhythm. In this study, we investigated changes in circadian BP and HR rhythm in SHR in various ages. Chronogram and cosinor method (Halberg et al. 1972) were used for the time series analyses.

\section{Methods}

We used male SHR and WKY obtained from the Institute for Experimental Animals, Tohoku University, Sendai, Japan. Three different age groups [5-6 week old WKY ( $n=$ $12)$ and SHR $(n=8), 19-22$ week old WKY $(n=8)$ and SHR $(n=10), 29-31$ week old WKY $(n=6)$ and SHR $(n=8)]$ were provided. The detail of the experimental procedures has been reported elsewhere (Imai et al. 1987). Briefly, under ether anaesthesia, a left femoral artery was cannulated using tapered PE-100 polyethylene tubing. The catheter was passed subcutaneously and brought out at the neck. The catheter was connected to a hydraulic, swivel-tethering system. Each rat was placed in an individual plastic metabolic cage kept in a room under conditions of constant temperature $\left(23^{\circ} \mathrm{C}\right)$, humidity $(60 \%)$ and light-dark cycle (light: 8:00 a.m. to $8: 00$ p.m., dark: $8: 00$ p.m. to $8: 00$ a.m.). The rats were housed in an isolated but not sound-proof room. They were continuously exposed to the noise from infusion pumps and rats nearby. The environmental conditions were the same for all experiments. To keep the arterial catheter patent, heparinized saline solution (100 $\mathrm{U} / \mathrm{ml}$ ) was infused continuously at the rate of $80 \mu \mathrm{l} / \mathrm{hr}$. The animals had a free access to a commercial diet and water. Blood pressure was recorded from the femoral artery catheter using a P23Ib Statham pressure transducer (Oxford, CA, USA) and straingauge amplifier 
(Model 1257 ; NEC-San-ei, Tokyo). The analogue signals of phasic pressure, mean BP and HR were fed to an analogue/digital converter (Mark 1; N.C.C., Tokyo). Digital signals of mean BP and HR from this converter were fed to a microcomputer (Model HP-9816; Hewlett-Packard, Fort Collins, CO, USA) every $4 \mathrm{sec}$ for $24 \mathrm{hr}$. At the end of each measurement period, the processed data were printed out. Mean values and standard deviation averaged for every $1-\mathrm{hr}$ and $24-\mathrm{hr}$ were calculated for each parameter. To eliminate the effect of anesthesia on circadian BP and HR rhythms as much as possible, we waited for at least $48 \mathrm{hr}$ after the operation to start recording.

\section{Time series analysis}

Two methods were used for time-series analyses. BP and HR were averaged in the same group for each clock hour. Mean values for each clock hour were plotted as a function of time. A chronogram was used to compare the difference in circadian changes of BP or HR between the groups. A correlation coefficient between BP and HR within each group was calculated to investigate the similarity in these circadian rhythms. We also used a single cosinor method for the statistical evaluation of parameters of circadian $\mathrm{BP}$ or $\mathrm{HR}$ rhythms. A cosine curve with a period for $24 \mathrm{hr}$ was fitted to the 24 time series data in the groups calculated above using the method of least squares. "F" statistics was then used to test the zero-amplitude hypothesis for the circadian rhythm. For a statistically validated rhythm $(p<0.05)$, the following parameters were estimated: the mesor (rhythm adjusted mean), the acrophase (the lag from a reference time to the crest time of the best fitting cosine curve) and the amplitude (one-half of the difference between the peak and nadir of BP or HR rhythms). Comparison of rhythm parameters was based on confidence intervals or regions as in the following. If the $1-\alpha$ confidence intervals (in the case of mesor comparison) or regions (in the case of amplitude-acrophase comparison) do not overlap, a statistically significant difference is indicated with $P<2 \alpha$ (Nelson et al. 1979) : i.e. $\alpha<0.05$ and thus $p<0.1$.

\section{Results}

As shown in Figs. 1 and 2, a clear nocturnal rise and diurnal fall of $\mathrm{BP}$ and $\mathrm{HR}$ was observed in all age groups of WKY. There was a significant positive correlation between $\mathrm{BP}$ and $\mathrm{HR}$ in each age group (Fig. 3, r $=0.817, p<0.01$ in young; $\mathrm{r}=0.630, p<0.01$ in adult: $\mathrm{r}=0.814, p<0.01$ in old).

Circadian rhythm of BP in 5-6 week old SHR was different from that of all age groups of WKY. BP demonstrated two peaks : one at around 4 to 5 A.M. and the other at around 10 A.M. The circadian HR rhythm, however, was almost identical to that of WKY. There was no significant correlation between BP and HR in 5-6 week old SHR (Fig. 3). In 19-22 week old SHR, BP was higher during day time and lower during the night time, indicating a circadian rhythm quite different from that of 5-6 week old SHR or WKY, while HR demonstrated a similar pattern seen in the control group (Fig. 2). Negative correlation between BP and HR was observed in 19-22 week old SHR (Fig. $3: \mathrm{r}=-0.586, p<0.01$ ). Higher BP during day time was also observed in 29-31 week old SHR. Since circadian HR rhythm in these rats was similar to that in other groups of rats, the relation between BP and HR in 29-31 week old SHR was also negative (Fig. 3; $\mathrm{r}=-0.749, p<0.01)$.

Cosinor parameters of $\mathrm{BP}$ and $\mathrm{HR}$ in WKY and SHR are summarized in 

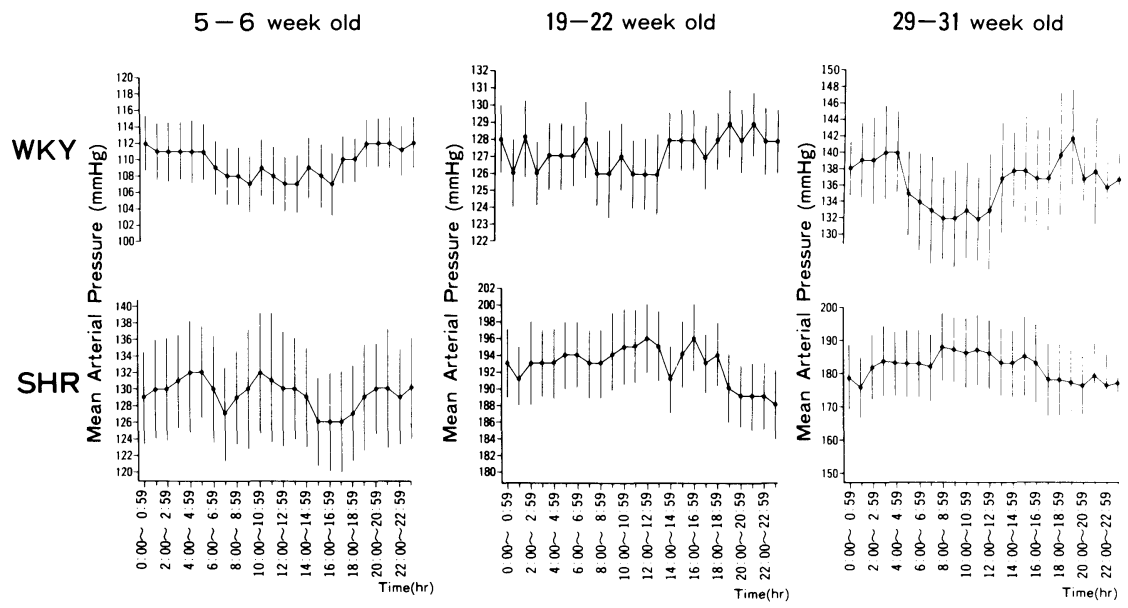

Fig. 1. Circadian variation of mean arterial pressure in Wistar-Kyoto rats (WKY) and spontaneously hypertensive rats (SHR). Left upper panel : 5-6 week old WKY $(n=12)$, left lower panel : 5-6 week old SHR $(n=8)$. Middle upper panel : 19-22 week old WKY $(n=8)$, middle lower panel: 19-22 week old SHR $(n=10)$. Right upper panel : 29-31 week old WKY $(n=6)$, right lower panel : 29-31 week old SHR $(n=8)$.

$5-6$ week old

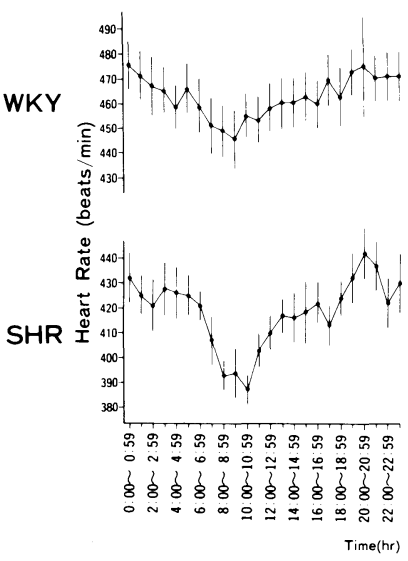

19-22 week old

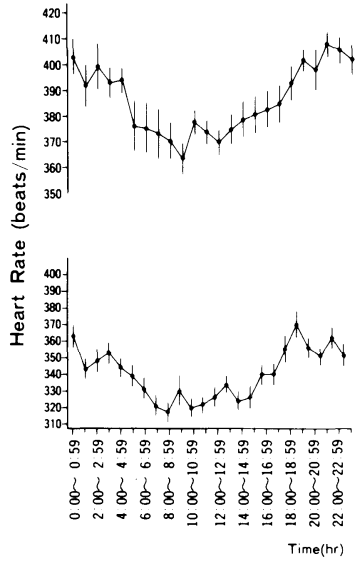

$29-31$ week old

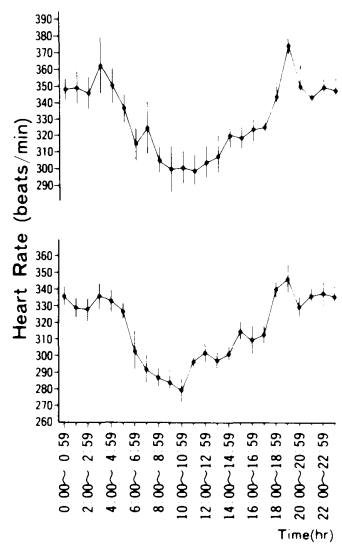

Fig. 2. Circadian variation of heart rate in WKY and SHR. Left upper panel : 5-6 week old WKY $(n=12)$, left lower panel : 5-6 week old SHR $(n=8)$. Middle upper panel : 19-22 week old WKY $(n=8)$, middle lower panel : 19 22 week old SHR $(n=10)$. Right upper panel : 29-31 week old WKY ( $n=$ $6)$, right lower panel : 29-31 week old SHR $(n=8)$.

Tables 1 and 2. Polar representation of amplitude and acrophase for BP and HR in WKY and SHR is shown in Fig. 4. As shown in Table 1 and Fig. 4, there was no significant difference in acrophase between $\mathrm{BP}$ and $\mathrm{HR}$ in three age groups of WKY. In the 5-6 week old SHR, acrophase of BP was significantly different 

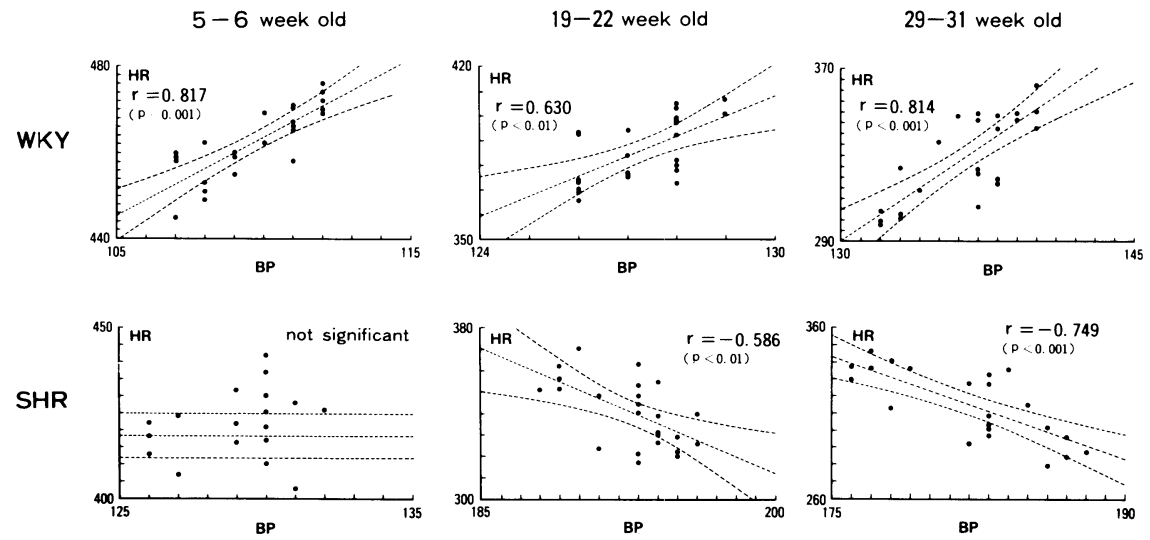

Fig. 3. Relationship between mean arterial pressure (BP) and heart rate (HR) in WKY and SHR. Left upper panel : 5-6 week WKY, left lower panel : 5-6 week old SHR. Middle upper panel : 19-22 week old WKY, middle lower panel : 19-22 week old SHR. Right upper panel : 29-31 week old WKY, right lower panel : 29-31 week old SHR.

TABLE 1. Cosinor summary of mean arterial pressure and heart rate in Wistar-Kyoto rats (WKY)

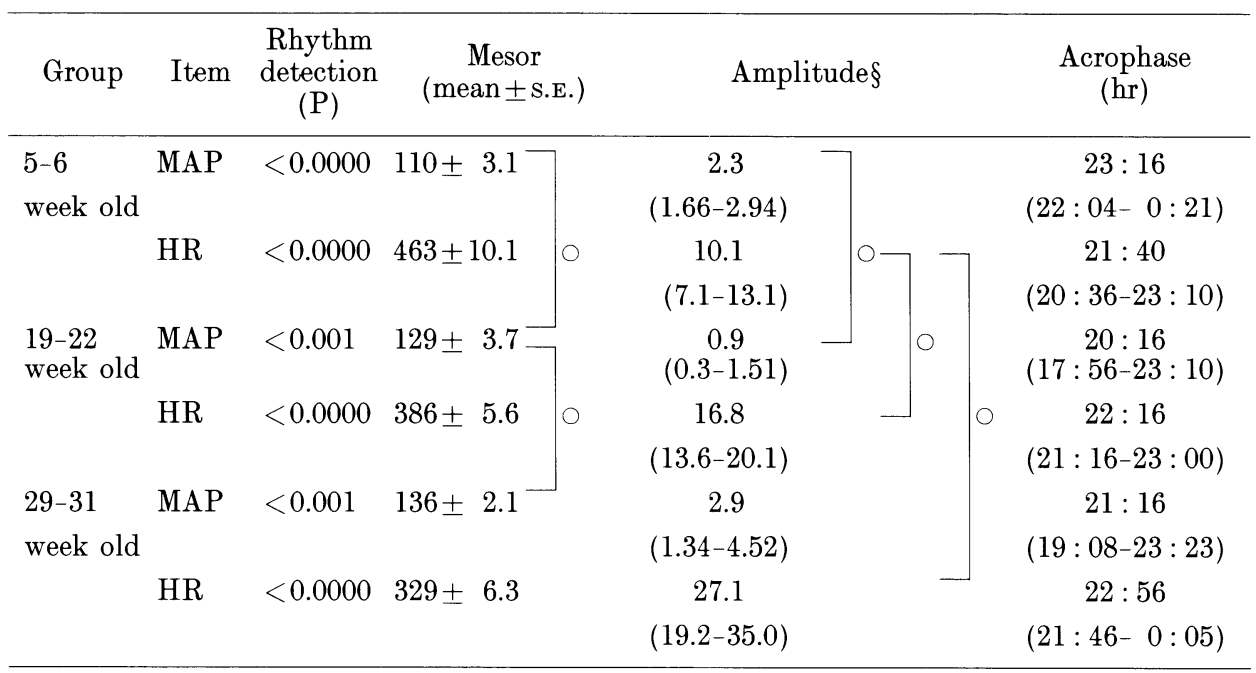

MAP, mean arterial pressure; HR, heart rate; $\mathrm{P}$, rhythm detection by zoroamplitude test, $\S \mathrm{mmHg}$ in $\mathrm{MAP}$ and beats $/ \mathrm{min}$ in $\mathrm{HR}$ and $95 \%$ confidence range in parenthesis. $\bigcirc, p<0.1$.

from that of HR $(6.9 \mathrm{hr}, p<0.1)$, the difference becoming even larger with the increase in age. Circadian BP rhythm of 19-22 week old SHR was the reverse of that of HR (11.0 hr, $p<0.1$, Fig. 4). Such relation was observed in 29-31 week old SHR as well $(11.1 \mathrm{hr}, p<0.1)$. Acrophase of HR in these three age groups of SHR was almost identical to that in WKY, indicating that circadian HR rhythm 
TABLE 2. Cosinor summary of mean arterial pressure and heart rate in spontaneously hypertensive rats ( $S H R$ )

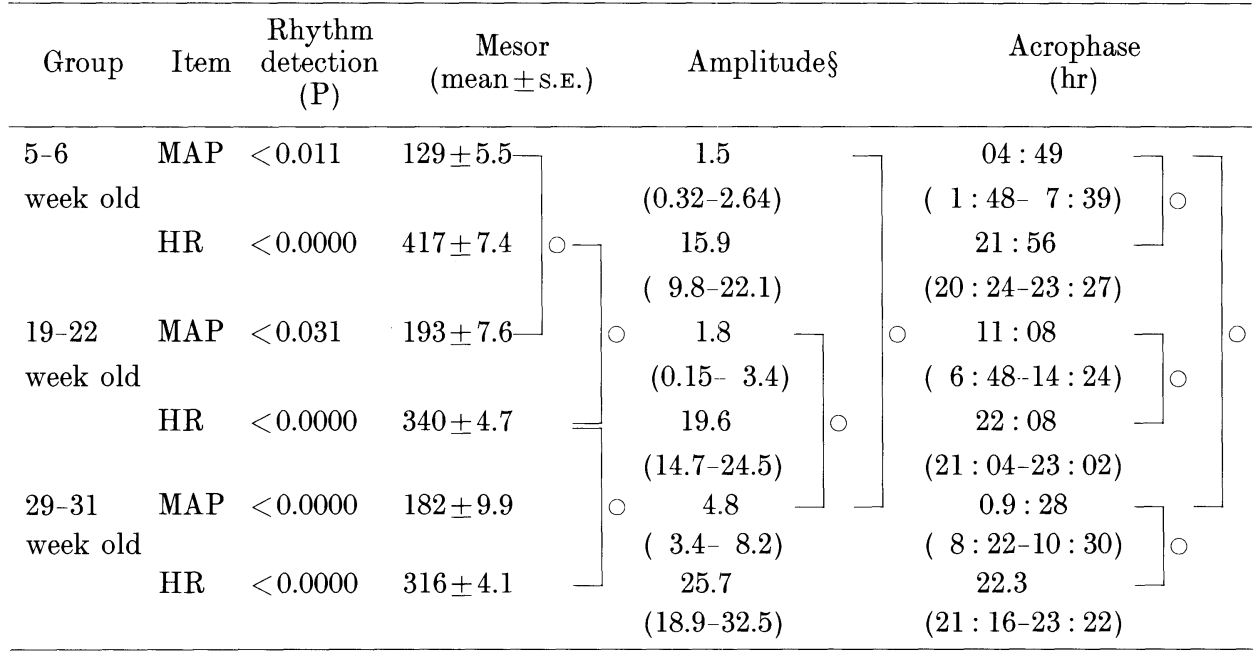

MAP, mean arterial pressure; $\mathrm{HR}$, heart rate; $\mathrm{P}$, rhythm detection by zeroamplitude test. $\$ \mathrm{mmHg}$ in $\mathrm{MAP}$ and beats $/ \mathrm{min}$ in $\mathrm{HR}$ and $95 \%$ confidence range in parenthesis. $\bigcirc, p<0.1$.

5-6 week old
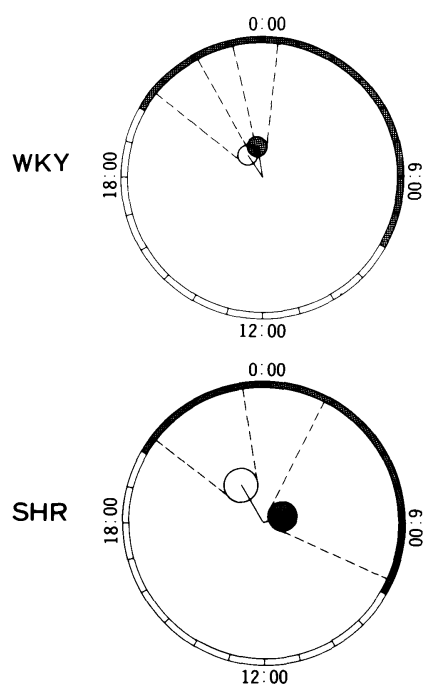

19-22 week old
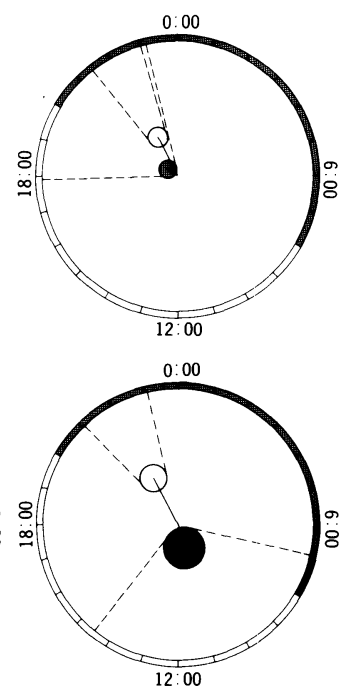

$29-31$ week old
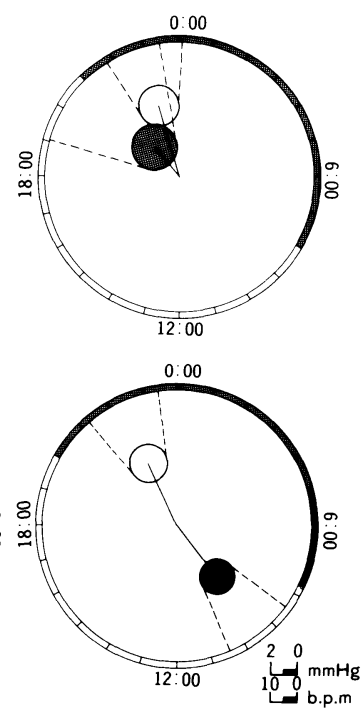

Fig. 4. Cosinor representation of amplitude and acrophase for mean arterial pressure (closed circles in each polar graph) and heart rate (open circles in each polar graph). Left upper panel : 5-6 week old WKY, left lower panel : 5-6 week old SHR. Middle upper panel : 19-22 week old WKY, middle lower panel : 19-22 week old SHR. Right upper panel : 29-31 week old WKY, right lower panel : 29-31 week old SHR. 
is affected by neither the strain nor the age of rats (Fig. 4).

\section{Discussion}

In the present study, we investigated circadian BP and HR rhythm in SHR and WKY. These strains were also studied for age dependent changes in circadian BP and HR rhythm. In each of WKY, a clear nocturnal rise and diurnal fall of $\mathrm{BP}$ and $\mathrm{HR}$ were observed. These two variables had good rhythm detection by a zero-amplitude test and there was no significant difference in acrophase between the two variables in each age group of WKY. In other words, the phase of circadian BP rhythm in WKY was in good agreement with circadian HR rhythm. On the other hand, in 5-6 week old SHR, a significant phase lag of circadian rhythm was observed between BP and HR, though both had good rhythm detection by the zero-amplitude test. This discrepancy became greater with the increase in age.

To assess the circadian BP rhythm, it is necessary to develop an efficient statistical and mathematical method. The cosinor method proved to be very useful for assessing physiological circadian rhythms including BP (Halberg et al. 1972 ; Nelson et al. 1979 ; Kawasaki et al. 1984). This method characterizes the rhythm of a population as well as the individual. There have been made some reports confirming circadian BP rhythm in rats (Su et al. 1985; Smith et al. 1987 ; Friberg et al. 1989), while the opposite cases of rats have also been reported (Norman et al. 1980). Halberg reported no phase lag of circadian rhythm between BP and HR in SHRSP, while an increase in amplitude of circadian BP rhythm preceding an actual rise in the BP mesor was observed in this strain (Halberg et al. 1979). There is, however, no report on the phase lag between circadian BP and circadian HR rhythm in SHR.

It is well known that cardiovascular activity is influenced by stress and physical activity (Rowlands et al. 1980). It has been reported that SHR are behaviorally more active than WKY (Hendley et al. 1985). Pasley et al. found that amplitude of circadian drinking rhythm in 12-16 week old SHR was larger than that in control rats, while there was no phase lag of circadian drinking rhythm between these two groups (Pasley et al. 1987). Kauker et al. reported that the circadian renal excretory rhythm such as urine volume, sodium or potassium excretion as well as water consumption in 11 week SHR was not different from that of age matched WKY (Kauker and Barr 1987). Thus, it is difficult to explain the change of circadian BP rhythm through that of circadian drinking rhythm or renal excretory rhythm. Since environmental factors such as temperature, humidity and light-dark cycle were the same for all groups of rats in the present experiment, it is unlikely that some external factors affect the circadian BP rhythm in SHR. It has been reported that circadian body temperature rhythm in 15-16 week old SHR is identical to that in control WKY (Lewis et al. 1986) and was in good agreement with the circadian HR rhythm. There- 
fore, circadian BP rhythm in SHR seems to be disturbed independently of other physiological rhythms such as body temperature, HR, and behavioral activity. Although we did not record electroencephalograms of the rats in the present study, circadian rhythm of the sleep-awake cycle must be similar in both strains, since drinking or behavioral activity is normally higher while awake and lower while asleep and circadian rhythm of drinking or behavioral activity was identical in both strains, as noted above (Kauker and Barr 1987; Pasley et al. 1987).

Although the mechanism responsible for the circadian BP rhythm is yet to be clarified, the circadian rhythm of sympathetic neural tone is suggested to play a major role in the regulation of circadian BP rhythm at least in humans (Akerstdt and Levi 1978; Prinz et al. 1979). This may indicate the disturbance of the periodicity of sympathetic neural tone relating to the regulation of circadian BP rhythm in SHR. On the other hand, the circadian HR rhythm, which has been postulated to be mediated by the periodicity of parasympathetic neural tone (Ewing et al. 1984), remained unchanged in SHR in the present study, indicating that the periodicity of parasympathetic neural tone in the SHR was not disturbed.

Inherent oscillator in the suprachiasmatic nucleus (SCN) of the anterior hypothalamus is reported to mediate some physiological rhythms such as the sleep-awake cycle, HR and plasma cortisol in rats (Moore 1983). Recent immunohistochemical investigations have shown several peptide-containing neurons in rat SCN, the largest population of which is vasopressin containing neurons (Moore 1983). There has been a report that a decrease in the number of vasopressinergic cells in the SCN of old rats may have some relation to the change in physiological circadian rhythm with aging (Rozendaal et al. 1987). The same phenomenon is also observed in humans (Stopa et al. 1984; Swaab et al. 1985). A reduced vasopressin content in the hypothalamus and brain stem has been confirmed in SHR (Rascher et al. 1982 ; Morris et al. 1983). It is possible that the disturbance of the central vasopressinergic system contributes, at least in part, to the change in circadian BP rhythm in SHR. Other neuronal mechanisms may also be considered responsible for the regulation of circadian BP rhythm, e.g., the catecholaminergic system. According to Lew, the circadian rhythm of norepinephrine in the hypothalamus is reversed in the genetically hypertensive NewZealand strain of rat (Lew 1976). In the present study, however, it is very difficult to determine the mechanism responsible for the dissociation of the circadian BP and HR rhythms in SHR.

In summary the present study demonstrated that a significant difference in acrophase between circadian BP and circadian HR rhythm was observed in SHR and this difference became greater with the increase in age. It is hypothesized that the mechanism responsible for development and maintenance of high $\mathrm{BP}$ in SHR is linked with disturbance of the sympathetic nervous system which controls $\mathrm{BP}$ periodicity. This interaction warrants further study. 


\section{References}

1) Akerstdt, T. \& Levi, L. (1978) Circadian rhythm in the secretion of cortisol, adrenaline and noradrenaline. Eur. J. Clin. Invest., 8, 57-58.

2) Bartter, F.C., Delea, C.S., Baker, W., Halberg, F. \& Lee, J.K. (1976) Chronobiology in the diagnosis and treatment of mesor-hypertension. Chronobiologia, 3, 199-213.

3) Ewing, D.J., Neilson, J.M. \& Travis, P. (1984) New method for assessing cardiac parasympathetic activity using 24 hour electrocardiograms. Br. Heart J., 52, 396402.

4) Friberg, P., Karlsson, B. \& Nordlander, M. (1989) Autonomic control of the diurnal variation in arterial blood pressure and heart rate in spontaneously hypertensive and Wistar-Kyoto rats. J. Hypertens., 7, 799-807

5) Halberg, F., Johnson, E.A., Nelson, E. \& Sothern, R. (1972) Autorhythmometry procedures for physiologic self-measurements and their analysis. Physiol. Teacher, 1, $1-11$.

6) Halberg, J., Halberg, E., Hayes, D.K. \& Bartter, F.C. (1979) Schedule shifts, life quality and quantity-modeled by murine blood pressure elevation and arthropod life span. Int. J. Chronobiol., 7, 17-64.

7) Hendley, E.D., Wessel, D.J., Atwater, D.G. \& Low, W.C. (1985) Age, sex and strain differences in activity and habituation in SHR and WKY rats. Physiol. Behav., 34, 379-383.

8) Imai, Y., Abe, K., Sasaki, S., Minami, N., Nobunaga, T., Sekino, H. \& Yoshinaga, K. (1987) Hypotensive and bradicardic effects of centrally administered vasopressin in stroke-prone spontaneously hypertensive rats. Hypertension, 10, 346-349.

9) Imai, Y., Abe, K., Sasaki, S., Minami, N., Nihei, M., Munakata, M., Murakani, O., Matsue, K., Sekino, H. \& Yoshinaga, K. (1988) Altered circadian blood pressure rhythm in patients with Cushing's syndrome. Hypertension, 12, 11-19.

10) Kauker, M.L. \& Barr, J.G. (1987) Circadian renal excretory rhythm in spontaneously hypertensive and Wistar-Kyoto rats. SD J. Med., 40, 25-28.

11) Kawasaki, T., Ueno, M., Uezono, K. \& Omae, T. (1984) The renin-angiotensin system and circadian rhythm of urine variables in normotensive and hypertensive subjects. Jpn. Circ. J., 48, 168-172.

12) Lew, GM. (1976) Circadian rhythm in blood pressure and norepinephrine in genetically hypertensive and normotensive rats. Gen. Pharmacol., 7, 35-40.

13) Lewis, S.J., Maccarrone, C. \& Jarrott, B. (1986) Modification of circadian body temperature rhythm of spontaneously hypertensive rat during and follwing cessation of continuous clonidine infusion. Brain Res., 385, 383-388.

14) Littler, W.A., Honour, A.J., Barter, R.D. \& Sleight, P. (1975) Sleep and blood pressure. Br. Med. J., 3, 346-348.

15) Mancia, G., Ferrari, A., Gregorini, L. \& Zanchetti, A. (1983) Blood pressure and heart rate variabilities in normotensive and hypertensive human beings. Circ. Res., 52, 96-104.

16) Mann, S., Altman, D.G., Raftery, E.B. \& Bannister, R. (1983) Circadian variation of blood pressure in autonomic failure. Circulation, 68, 477-483.

17) Michael, W., Millar, C., Stewart, M. \& Raftery, E.B. (1978) Blood pressure circadian rhythm in essential hypertension. Clin. Sci. Mol. Med., 55, 391-393.

18) Moore, R.Y. (1983) Organization and function of a central nervous system circadian oscillator: The suprachiasmatic hypothalamic nucleus. Fed. Proc., 42, 2783-2789.

19) Morris, M., Keller, M. \& Sundberg, D.K. (1983) Change in paraventricular vasopressin and oxytocin during the development of spontaneous hypertension. Hypertension, 5, 476-481.

20) Nelson, W., Yong, Y.L., Lee, J.K. \& Halberg, F. (1979) Methods for cosinor- 
rhythmometry. Chronobiologia, 6, 305-323.

21) Norman, R.A., Jr., Coleman, T.G. \& Dent, A.C. (1980) Pseudohypertension in sinoaortic denervated rats. Clin. Sci., 59, 303s-306s.

22) Pasley, J.N., Pomell, E.W. \& Halberg, F. (1987) Strain differences in circadian drinking behaviors of ethanol and water in rats. Prog. Clin. Biol. Res., 227B, 467471.

23) Prinz, P.N., Halter, J., Benedetti, C. \& Raskind, M. (1979) Circadian variation of plasma cathecholamines in young and old men in relation to rapid eye movement and slow wave sleep. J. Clin. Endocr., 49, 300-304.

24) Rascher, W., Lang, R.E., Unger, T., Ganten, D. \& Gross, F. (1982) Vasopressin in brain of spontaneously hypertensive rats. Am. J. Physiol., 242, H496-H499.

25) Redman, C.W.G., Beilin, L.J. \& Bonnar, J. (1976) Reversed diurnal blood pressure rhythm in hypertensive pregnancies. Clin. Sci. Mol. Med., 51, 687s-689s.

26) Richards, A.M., Nicholls, M.G., Espiner, E.A. \& Hinton, D. (1986) Diurnal patterns of blood pressure, heart rate and vasoactive hormones in normal man. Clin. Exp. Hypertens., A8, 153-166.

27) Richardson, D.W., Honour, A.J., Fenton, G.W. \& Pickering, G.W. (1964) Variations in arterial pressure throughout the day and night. Clin. Sci., 26, 445-460.

28) Rowlands, D.B., Stalland, T.J., Watson, R.D.S. \& Littler, W.A. (1980) The influence of physical activity on arterial pressure during ambulatory recordings in man. Clin. Sci., 58, 115-117.

29) Rozendaal, B., VanGool, W.A., Swaab, D.F., Hoogendijek, J.E. \& Mirmiran, M. (1987) Change in vasopressin cells of the rat suprachiasmatic nucleus with aging. Brain Res., 409, 259-264.

30) Smith, T.L., Coleman, T.G., Staneck, K.A. \& Murphy, W.R. (1987) Hemodynamic monitoring for $24 \mathrm{hr}$ in unrestrained rats. Am. J. Physiol., 253, H1335-H1341.

31) Stopa, E.G., King, J.C. \& Shoene, W.C. (1984) Human brain contains vasopressin and vasoactive intestinal polypeptide neuronal subpopulation in the suprachiasmatic region. Brain Res., 297, 159-163.

32) Su, D.F., Cerutti, C., Barres, C. \& Sassard, J. (1985) Computer analysis of cardiovascular activity in conscious unrestricted hypertensive rats of the Lyon strain. Clin. Exp. Hypertens., A7, 413-416.

33) Swaab, D.F., Fliers, E. \& Partiman, T.S. (1985) The suprachiasmatic nucleus of the human brain in relation to sex, age and senile dementia. Brain Res., 342, 37-44.

34) Weber, M.A., Drayer, J.I., Wyle, F.A. \& DeYong, J.L. (1982) Reproducibility of the whole-day blood pressure pattern in essential hypertension. Clin. Exp. Hypertens., A4, 1377-1390. 